\title{
Proteomic profile of dormancy within Staphylococcus epidermidis biofilms using iTRAQ and label-free strategies
}

\author{
Virginia Carvalhais • Nuno Cerca - Manuel Vilanova • \\ Rui Vitorino
}

Received: 27 August 2014 / Revised: 16 December 2014 / Accepted: 25 January 2015 /Published online: 12 February 2015

(C) Springer-Verlag Berlin Heidelberg 2015

\begin{abstract}
Staphylococcus epidermidis is an important nosocomial bacterium among carriers of indwelling medical devices, since it has a strong ability to form biofilms. The presence of dormant bacteria within a biofilm is one of the factors that contribute to biofilm antibiotic tolerance and immune evasion. Here, we provide a detailed characterization of the quantitative proteomic profile of $S$. epidermidis biofilms with different proportions of dormant bacteria. A total of 427 and 409 proteins were identified by label-free and label-based quantitative methodologies, respectively. From these, 29 proteins were found to be differentially expressed between $S$. epidermidis biofilms with prevented and induced dormancy. Proteins overexpressed in S. epidermidis with prevented dormancy were associated with ribosome synthesis pathway, which reflects the metabolic state of dormant bacteria. In the
\end{abstract}

Electronic supplementary material The online version of this article (doi:10.1007/s00253-015-6434-3) contains supplementary material, which is available to authorized users.

V. Carvalhais $\cdot$ N. Cerca

Centre of Biological Engineering (CEB), Laboratory of Research in Biofilms Rosário Oliveira (LIBRO), University of Minho, Campus de Gualtar, 4710-057 Braga, Portugal

V. Carvalhais $\cdot R$. Vitorino $(\square)$

QOPNA, Mass Spectrometry Center, Department of Chemistry,

University of Aveiro, 3810-193 Aveiro, Portugal

e-mail: rvitorino@ua.pt

M. Vilanova

Instituto de Biologia Molecular e Celular (IBMC), Rua do Campo

Alegre 83, Porto, Portugal

M. Vilanova

Instituto de Ciências Biomédicas Abel Salazar (ICBAS), University of Porto, Rua de Rua de Jorge Viterbo Ferreira 228,

4050-313 Porto, Portugal

R. Vitorino

University of Aveiro, Aveiro, Portugal opposite, underexpressed proteins were related to catalytic activity and ion binding, with involvement in purine, arginine, and proline metabolism. Additionally, GTPase activity seems to be enhanced in S. epidermidis biofilm with induced dormancy. The role of magnesium in dormancy modulation was further investigated with bioinformatics tool based in predicted interactions. The main molecular function of proteins, which strongly interact with magnesium, was nucleic acid binding. Different proteomic strategies allowed to obtain similar results and evidenced that prevented dormancy led to an expression of a markedly different repertoire of proteins in comparison to the one of dormant biofilms.

Keywords Carbonylation · Dormancy · emPAI · iTRAQ · Quantitative proteomics · Staphylococcus epidermidis biofilm

\section{Introduction}

Abiotic and biotic surfaces are prone to bacterial attachment and colonization (Otto 2013). Staphylococcus epidermidis is among the most common bacteria which form biofilms in the surface of medical devices (Otto 2014). S. epidermidis biofilms comprise a matrix composed of several extracellular polymeric substances, such as polyssacharides (Donlan 2001; Sadovskaya et al. 2005), proteins (Cucarella et al. 2001; Lasa and Penades 2006; Rohde et al. 2005), extracellular teichoic acids (Sadovskaya et al. 2004), and extracellular DNA (Qin et al. 2007). The biofilm mode of growth has important clinical implications such as higher tolerance to antibiotics (Cerca et al. 2005) and resistance to the innate immune system (Cerca et al. 2006; Fey 2010; Vuong et al. 2004). Biofilms also contain cells with a wide range of metabolic activities (HallStoodley et al. 2004). It has been reported that bacterial cells 
in the deeper layers of the biofilms are less active than the ones in the upper layers, although they are still viable but with a slower growth rate (Stewart and Franklin 2008; Williamson et al. 2012). Nowadays, despite controversial, persistence and dormancy are one of the most discussed topics in biofilm field (Balaban et al. 2013; Orman and Brynildsen 2013). Dormant and persister cells are associated with higher tolerance to antibiotics without undergoing genetic changes (Lewis 2007) and may determine its inflammatory profile (Cerca et al. 2011, 2014). However, recent findings suggest that persistence is not directly associated with dormancy (Orman and Brynildsen 2013; Wakamoto et al. 2013). Dormant bacteria are defined as bacteria which persist without division for extended periods, since they present a reversible low metabolic activity (Kaprelyants et al. 1993; Lewis 2007). Despite mechanisms behind this condition remain unknown, several stress factors may induce the formation of dormant bacteria, such as DNA damaging agents, high temperatures, low $\mathrm{pH}$, and membrane-acting agents (Dorr et al. 2010). Previously, we developed an in vitro model to modulate dormancy within S. epidermidis biofilms (Cerca et al. 2011). According to this model, the culture medium acidification due to glucose metabolism was responsible for inducing bacteria to enter into a dormant state (Cerca et al. 2011). In contrast, dormancy was prevented by adding magnesium $\left(\mathrm{Mg}^{2+}\right)$ to the culture medium (Cerca et al. 2011). Recently, we determined the transcriptomic profile of $S$. epidermidis biofilms with different proportions of dormant bacteria which confirmed that the translation process was related to the proportion of dormant bacteria (Carvalhais et al. 2014). Transcriptomic data analysis also suggested that oxidation-reduction processes were associated with dormancy. However, the expression level of a gene does not necessarily indicate the amount of protein produced, nor its biological activity (Zhang et al. 2010). Similar to other bacteria (reviewed by de Sousa et al. (2009)), S. epidermidis transcripts and proteins showed a modest correlation (Carvalhais et al. 2015), which suggests that transcript regulation and protein modifications may determine the biological importance of a gene (Cain et al. 2013; Guell et al. 2011; Straub 2011).

Currently, proteomic tools allow simultaneously the identification and comparison of protein expression in different pathophysiological conditions. Quantitative proteomic analysis based on mass spectrometry may be achieved, among others, by stable isotope labeling, such as isobaric tags for relative and absolute quantitation (iTRAQ) or by label-free spectral counting, such as exponentially modified protein abundance index (emPAI) (Abdallah et al. 2012). In one experimental setup, iTRAQ has the capability of multiplexing up to eight samples (Ross et al. 2004) where proteolytic peptides are chemically labeled with amine-specific isobaric tags. Then, upon induced fragmentation in the mass spectrometer, peptides yield different reporter ions in $113-121 \mathrm{~m} / \mathrm{z}$ range (in the case of an 8-plex experiment) (Ross et al. 2004). Alternatively, the relative protein abundance (emPAI) offers label-free relative quantitation of a protein in a complex sample, based on protein coverage by the peptide matches which is proportional to the protein abundance in a cell (Ishihama et al. 2005). The ability to accurately quantify proteins by label-free spectral counting depends on the number of spectra obtained and the sample coverage (Abdallah et al. 2012). Thus, apart from relative quantitation, label-free also allows a qualitative analysis based on the number of identified proteins. Conversely, iTRAQ methodology do not allow to identify unique proteins, since the protein ratio is only calculated when the protein is present in both tested conditions.

The purpose of our study was to describe quantitative profile of $S$. epidermidis biofilms with induced and prevented dormancy, using label-free and label-based proteomic approaches. To achieve this, total proteins from $S$. epidermidis biofilms with different proportion of dormant cells were quantified by iTRAQ coupled with 2DLC-MS/MS and by SDSPAGE-LC-MS/MS (GeLC-MS/MS).

\section{Materials and methods}

\section{Biofilm growth conditions}

One colony of S. epidermidis 9142 (isolated from blood culture (Mack et al. 1992)) (collection number 18857 at DSM, Braunschweig, Germany) was inoculated in tryptic soy broth (TSB) (LiofilChem, Roseto Degli Abruzzi, Italy) and incubated at $37{ }^{\circ} \mathrm{C}$ in an orbital shaker at $120 \mathrm{rpm}$ for $18 \mathrm{~h}$. The overnight culture was adjusted to an optical density at $640 \mathrm{~nm}$ of $0.250( \pm 0.05)$ with TSB, and $10 \mu \mathrm{L}$ of the suspension was transferred into a 24-well plate (Orange Scientific, Braine-l'Alleud, Belgium) containing $1 \mathrm{~mL}$ of TSB supplemented with $0.4 \%$ glucose $(v / v)$ (TSB $0.4 \%$ G) (Fisher Scientific, Waltham, MA, USA) or TSB $0.4 \% \mathrm{G}$ enriched with $20 \mathrm{mM}$ magnesium chloride $\left(\mathrm{MgCl}_{2}\right)$ (Merck, Darmstadt, Germany), to form biofilms. The culture plates were then incubated at $37^{\circ} \mathrm{C}$ in an orbital shaker at $120 \mathrm{rpm}$ for $24 \mathrm{~h}$. After this period, the culture medium was removed and replaced by fresh TSB supplemented with $1 \%$ glucose $(v / v)(1 \% \mathrm{G})$ or TSB $1 \% \mathrm{G}$ containing $20 \mathrm{mM} \mathrm{MgCl} 2\left(1 \% \mathrm{G}+\mathrm{Mg}^{2+}\right)$. Biofilms were then allowed to grow in these same conditions for an additional $24 \mathrm{~h}$. Next, biofilm culture medium was removed and biofilms were washed two times with phosphate-buffered saline (PBS). Then, bacteria within the biofilms were resuspended in $1 \mathrm{~mL}$ of PBS. Biofilm dormancy was assessed as previously described (Carvalhais et al. 2014; Cerca et al. 2011). Briefly, the number of CFU/mL in each biofilm growth condition was determined using the spread plate method in trypticase soy agar (LiofilChem). A 
reduction of about one log difference is typically expected in similarly grown biofilms without addition of $\mathrm{Mg}^{2+}$.

\section{Preparation of protein extracts}

For total protein extraction, biofilms were directly scrapped and resuspended with detergent extraction buffer, $25 \mathrm{mM}$ Tris- $\mathrm{HCl}(\mathrm{pH}=7.2)$ (Pharmacia Biotech, Uppsala, Sweden), 10 mM CHAPS (Sigma-Aldrich, St. Louis, MO, USA), 0.5 M $\mathrm{NaCl}$ (VWR, Radnor, PA, USA), $5 \%$ glycerol (Sigma-Aldrich), and $1 \mathrm{mM}$ PMSF (Sigma-Aldrich). Then, mechanical lysis was performed in a bead beating using glass beads of $0.1 \mathrm{~mm}$ (Sigma-Aldrich) in a FastPrep ${ }^{\circledR}$ cell disruptor (BIO 101, ThermoElectron Corporation) (three cycles of $30 \mathrm{~s}$ and $6.5 \mathrm{~m} / \mathrm{s}$ ). After lysis, cell debris were removed by centrifugation $\left(15,000 \mathrm{~g}\right.$ for $15 \mathrm{~min}$ at $\left.4{ }^{\circ} \mathrm{C}\right)$. Then, proteins were precipitated with $20 \%$ of TCA-cold acetone and quantified using the RC-DC assay (Bio-Rad, Hercules, CA, USA), following the manufacturer's instructions.

Gel electrophoresis (SDS-PAGE), in-gel protein digestion, and protein identification

Forty micrograms of protein were incubated with SDS $10 \%$ $(w / v)$ (USB Corporation, Cleveland, OH, USA), 0.5 M Tris$\mathrm{HCl}$ (pH 6.8), glycerol, mercaptoethanol (Sigma-Aldrich), and bromophenol blue $(w / v)$ for $5 \mathrm{~min}$ at $100{ }^{\circ} \mathrm{C}$. Then, samples were loaded on a SDS-PAGE and proteins were separated at a constant voltage $(150 \mathrm{~V})$ for $50 \mathrm{~min}$. The gel was stained with colloidal Coomassie G-250, and entire gel lanes were manually excised into 16 gel slices for in-gel digestion with trypsin TPCK treated (ABSciex, Framingham, MA, USA). Peptide extraction was made with $10 \%$ formic acid/ acetonitrile acid. Peptides were dried in a SpeedVac (Thermo Savant, Thermo Scientific) and were dissolved in $5 \%$ acetonitrile (MS grade, VWR), $0.1 \%$ formic acid (Fluka Analytical, Sigma-Aldrich) and $0.1 \%$ trifluoroacetic acid (Sigma-Aldrich). Separation of tryptic peptides by nanoHPLC was performed on the module separation Ultimate 3000 (Dionex, Thermo Fisher Scientific) using a capillary column (Pepmap100 C18; 3- $\mu \mathrm{m}$ particle size, 0.75- $\mu \mathrm{m}$ internal diameter, $15 \mathrm{~cm}$ in length). A gradient of solvent A (water/ acetonitrile/trifluoroacetic acid $(98: 2: 0.05, v / v / v))$ to solvent B (water/acetonitrile/trifluoroacetic acid (10:90:0.045, v/v/v)) was used. The separation of $2 \mu \mathrm{g} / \mu \mathrm{L}$ sample was performed using a linear gradient (5-50 \% B for $30 \mathrm{~min}, 50-70 \% \mathrm{~B}$ for $10 \mathrm{~min}$, and $70-5 \%$ A for $5 \mathrm{~min}$ ) with a flow rate of $0.3 \mu \mathrm{L} /$ $\min$. The eluted peptides were mixed with a continuous flow of $\alpha$-CHCA matrix solution $(270 \mathrm{~nL} / \mathrm{min}, 2 \mathrm{mg} / \mathrm{mL}$ in $70 \%$ $\mathrm{ACN} / 0.1 \%$ TFA and internal standard Glu-Fib at $15 \mathrm{ftmol}$ ) and were applied directly on a MALDI plate in 20-s fractions using an automatic fraction collector Probot (Dionex). Mass spectra were obtained on a matrix-assisted laser desorption/ ionization-time-of-flight (MALDI-TOF/TOF) mass spectrometer (4800 Proteomics Analyzer, Applied Biosystems, Foster City, CA, USA) in the positive ion reflector mode and obtained in the mass range from 700 to $4500 \mathrm{Da}$ with 900 laser shots. A fragmentation voltage of $2 \mathrm{kV}$ was used throughout the automated runs. The spectra were processed and analyzed by the T2S (v1.0, Matrix Science Ltd.) and submitted in Mascot software (v.2.1.0.4, Matrix Science Ltd.) for protein/peptide identification based on MS/MS data using the following criteria: trypsin as enzyme, a maximum of two missed cleavages, mass tolerances of $40 \mathrm{ppm}$ for peptide precursors, and mass tolerance of 0.6 Da was set for fragment ions. The local false discovery rate (FDR) was calculated by searching the spectra against SwissProt (Firmicutes, release date February 6, 2013) decoy database (false-positive peptides / (false-positive peptides + total peptides $) \times 100$ ). Protein identification based on a single peptide hit had a minimum individual score of $29(95 \%)$ and a minimum sequence tag of four amino acids (five consecutive peaks in the MS/MS spectrum). Quantification was performed using exponentially modified protein abundance index (emPAI). Gene ontology (GO) (Ashburner et al. 2000) terms and Kyoto Encyclopedia of Genes and Genomes (KEGG) (Kanehisa et al. 2004) pathways in each condition were determined using Search Tool for the Retrieval of Interacting Genes/Proteins (STRING) database (version 9.1) (accessed at December 10, 2014) (Franceschini et al. 2013). Independent replicates were performed with 22 pools of $S$. epidermidis biofilms, for each condition.

\section{iTRAQ labeling}

An in-solution digestion was performed for iTRAQ labeling, as previously described (Alves et al. 2013). Briefly, $100 \mu \mathrm{g}$ of protein was precipitated with 6 volumes of cold acetone at $-20{ }^{\circ} \mathrm{C}$ for $3 \mathrm{~h}$. After sample centrifugation and acetone decantation, pellets were resuspended with triethylammonium bicarbonate buffer (TEAB) (0.1 M, pH 8.5) (Sigma-Aldrich) and $2 \%$ SDS to achieve a final concentration of $0.05 \%$. Samples were then reduced with $50 \mathrm{mM}$ tris(2carboxyethyl)phosphine (TCEP) (Sigma-Aldrich) for $1 \mathrm{~h}$ at $37{ }^{\circ} \mathrm{C}$ with agitation. Then, samples were alkylated with $10 \mathrm{mM}$ S-methyl methanethiosulfonate (MMTS) (SigmaAldrich) for $10 \mathrm{~min}$ at room temperature with agitation. Trypsin was added to each sample, and the digestion was performed for $18 \mathrm{~h}$ at $37{ }^{\circ} \mathrm{C}$. Digested sample peptides were subsequently labeled with the iTRAQ ${ }^{\circledR}$ reagent 8 -plex (ABSciex) following the protocol provided by the manufacturer. Briefly, labels were reconstituted in $60 \%$ isopropanol, added to each sample peptides and incubated for $2 \mathrm{~h}$ at room temperature with agitation. Peptides from $S$. epidermidis biofilms with induced dormancy $(1 \% \mathrm{G})$ were labeled with 119 isobaric reagent and peptides from biofilms with 
prevented dormancy $\left(1 \% \mathrm{G}+\mathrm{Mg}^{2+}\right)$ were labeled with 121 isobaric reagent. The reaction was stopped by adding water and acidification with formic acid. Labeled samples were then combined and dried in SpeedVac.

Then, labeled peptides were separated from an adapted multidimensional LC approach, as described by Vitorino et al., based on high $\mathrm{pH}$ for first dimension peptide chromatography with a C18 reverse-phase HPLC column and acidic $\mathrm{pH}$ for second one (Vitorino et al. 2012). Thus, sample loading was performed at $200 \mu \mathrm{L} / \mathrm{min}$ with buffers (A) $2 \%$ ammonium hydroxide and $0.014 \%$ formic acid in water, $\mathrm{pH} 10$ and (B) $2 \%$ ammonium hydroxide and $90 \%$ acetonitrile (ACN) in water, $\mathrm{pH} 10$. After $5 \mathrm{~min}$ of sample loading and washing, peptide fractionation was performed with linear gradient to $70 \%$ B over $85 \mathrm{~min}$. Sixty fractions were collected, dried in a SpeedVac, and resuspended in $5 \% \mathrm{ACN}$ and $0.1 \%$ TFA. Collected fractions were then separated by LC. Briefly, peptides loaded onto a C18 pre-column (5-mm particle size, $5 \mathrm{~mm}$; Dionex) connected to an RP column PepMap100 C18 (150 $\mathrm{mm} \times 75-\mathrm{mm}$ i.d., 3-mm particle size). The flow rate was set at $300 \mathrm{~nL} / \mathrm{min}$. The mobile phases A and B were $2 \%$ $\mathrm{ACN}$ and $0.05 \%$ TFA in water, and $90 \% \mathrm{ACN}$ with $0.045 \%$ TFA in water, respectively. The gradient started at $10 \mathrm{~min}$ and ramped to $60 \% \mathrm{~B}$ till $50 \mathrm{~min}$ and $100 \% \mathrm{~B}$ at $55 \mathrm{~min}$ and retained at $100 \% \mathrm{~B}$ till $65 \mathrm{~min}$. The separation was monitored at $214 \mathrm{~nm}$ using a UV detector (Dionex/LC Packings). Using the microcollector Probot (Dionex/LC Packings) and after a lag time of $5 \mathrm{~min}$, peptides eluting from the capillary column were mixed with a continuous flow of $\alpha$-CHCA matrix solution (in internal standard Glu-Fib) and were directly deposited onto the LC-MALDI plates. The spectra were generated and processed with 4800 MALDI-TOF/TOF. Protein identification based on MS/MS data were performed with ProteinPilot ${ }^{\mathrm{TM}}$ software (v.4.04, AB Sciex) using Paragon search method. SwissProt from S. epidermidis strain RP62A (Firmicutes, release date February 6, 2013) was used as protein database. Default search parameters used were as follows: trypsin as the digestion enzyme with two missed cleavages, 40-ppm tolerance, carbamidomethyl modification on cysteine residue, iTRAQ 8-plex modification of $\mathrm{N}$-terminal and lysine peptide residues as fixed modification. Additionally, biological modifications with emphasis on methionine oxidation, deamidation and iTRAQ 8-plex modification of tyrosine residue and deamidation were considered variable modifications. Bias correction was applied, and proteins were identified with a confidence level of $95 \%$. Proteins were found to be differentially expressed when $p<0.05$ (FDR-corrected). Proteins were considered overexpressed in prevented dormancy when the $\mathrm{ITRAQ}$ ratio was above 1 and underexpressed when iTRAQ ratio was lower than 1 . GO and KEGG were determined using STRING as described in "Gel electrophoresis (SDS-PAGE), in-gel protein digestion, and protein identification" section.
Immunodetection and identification of oxidized proteins

Carbonylated proteins were assayed as previously described (Conrad et al. 2001; Robinson et al. 1999). Briefly, whole cell proteins from both conditions were separated by isoelectric focusing with IPG strips nonlinear $\mathrm{pH}$ 3-10 gradient (GE Healthcare, Buckinghamshire, UK). Then, each strip was incubated with $12 \%$ SDS for $30 \mathrm{~min}$ at room temperature and derivatized with $20 \mathrm{mM}$ 2,4-dinitrophenylhydrazine (DNPH) (Acros Organics, Geel, Belgium) in $10 \%$ TFA for $30 \mathrm{~min}$ at room temperature, in the dark. The reaction was stopped with equilibration buffer $(2 \%(w / v)$ SDS, $6 \mathrm{M}$ urea, $30 \%$ glycerol, $0.05 \mathrm{M}$ Tris $-\mathrm{HCl}(\mathrm{pH} 8.8)$, and $20 \mathrm{mg} / \mathrm{mL}$ DTT for $30 \mathrm{~min}$ at room temperature. The second dimension was obtained by SDS-PAGE. Then, gels were stained with colloidal Coomassie G-250 or gels were transferred onto a nitrocellulose membrane. Immunodetection was performed using anti2,4-dinitrophenol (DNP) antibody (Merck) and detected by enhanced chemiluminescence ECL (Amersham Pharmacia Biotech) according to the manufacturer's procedure. Images were recorded using X-ray films (GE Healthcare) and the films and the gels were scanned in Molecular Imager Gel Doc $\mathrm{XR}^{+}$system (Bio-Rad) and analyzed with QuantityOne software (v. 4.6.9, Bio-Rad). Protein spots reactive to antiDNP antibody were manually excised, and an in-gel digestion was performed as described in "Gel electrophoresis (SDSPAGE), in-gel protein digestion, and protein identification" section. Peptides were resuspended and directly deposited onto 384-well MALDI plates (Applied Biosystems) with $\alpha$ CHCA matrix solution. $\alpha$-CHCA matrix solution was prepared by diluting $2.5 \mathrm{mg} / \mathrm{mL}$ of $\alpha$-CHCA in ACN $70 \% /$ TFA $0.3 \%$. Experiments were carried in duplicate. Peptide mass spectra were obtained as referred in "Gel electrophoresis (SDS-PAGE), in-gel protein digestion, and protein identification" section. Mass spectra were processed and analyzed by the Global Protein Server (GPS) Workstation (Applied Biosystems). Searches were performed against the SwissProt protein database for Firmicutes (release date February 6, 2013). The database search parameters were as follows: mass tolerance of $40 \mathrm{ppm}$ for precursor ions and 0.4 Da for fragment ions, and trypsin digestion with two missed cleavages. Protein identity was accepted at the $95 \%$ confidence level.

\section{Results}

The work reported here represents a multiapproach to determine the protein changes in dormancy within $S$. epidermidis biofilms, using a previously described model based in $\mathrm{Mg}^{2+}$ (Cerca et al. 2011). As expected (Carvalhais et al. 2014; Cerca et al. 2011), the number of culturable bacteria in biofilms with induced dormancy $(1 \% \mathrm{G})$ was lower than the number of CFU per milliliter of biofilms with prevented dormancy 
$\left(1 \% \mathrm{G}+\mathrm{Mg}^{2+}\right)$, as shown in the supplementary material (Fig. S1).

\section{Label-free data analysis}

After GeLC-MS/MS separation and identification of proteins from induced $(1 \% \mathrm{G})$ and prevented $\left(1 \% \mathrm{G}+\mathrm{Mg}^{2+}\right)$ dormancy in $S$. epidermidis biofilms, label-free qualitative and relative quantitation analysis were performed from two independent replicates. For qualitative analyzes, Venn diagram was generated with identified proteins in each condition, using Venny tool from BioinfoGP (Oliveros 2007) (Fig. 1). A total of 378 and 387 proteins (FDR $<5 \%$ ) were identified in induced and prevented dormancy, respectively. Statistically significant GO terms (including biological processes and molecular functions) for proteins found in each condition are detailed in Fig. 2. Despite no major differences were found, cell wall organization or biogenesis (GO:0071554) was a biological process only found statistically significant in $S$. epidermidis biofilms with induced dormancy (including the MurC, Ddl, MurG, LtaS, Atl, DltC, MurA1, FemB, FemA, DltA, MurB, FemX, and GlmU proteins). GTPase activity (GO:0003924) was a molecular function only found statistically significant in S. epidermidis biofilms with induced dormancy. Examples of such proteins included SERP0696, InfB, PrfC, Obg, MnmE, UreG, Era, LepA, FtsZ, FusA, and EF-Tu. Among them, only seven proteins were common to the proteome of $S$. epidermidis biofilm with prevented dormancy. Interestingly, these proteins are involved in the nucleotide metabolic process and present ion-binding activity. Furthermore, cofactor metabolic process (GO:0051186) was a biological process only significant in prevented dormancy. Additionally, hydrolase activity, acting on carbon-nitrogen bonds (GO:0016810) and lyase activity (GO:0016829) were molecular function categories only found significant in prevented dormancy. Among all the proteins identified in both conditions, only 40 proteins were exclusively assigned to dormancy in S. epidermidis biofilms (Fig. 1; Table S1). Conversely, only 49 proteins were exclusively assigned to $S$. epidermidis

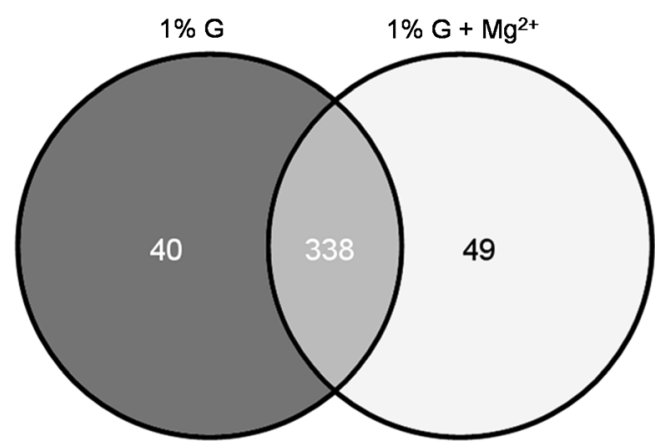

Fig. 1 Venn diagram summarizing the overlap between proteins identified by label-free methodology in S. epidermidis biofilms with prevented $\left(1 \% \mathrm{G}+\mathrm{Mg}^{2+}\right)$ or induced $(1 \% \mathrm{G})$ dormancy biofilms with prevented dormancy (Fig. 1; Table S2). Thus, Fig. 3 shows the three most represented biological processes and molecular functions in unique proteins found in each condition. Main differences were found in molecular function annotations. Herein, GTPase activity (GO:0003924), structural constituent of ribosome (GO:0003735), and structural molecule activity (GO:0005198) were categories predominant in induced dormancy. Conversely, DNA binding (GO:0003677) (including AddA, Rex, GreA, LexA, GrlA, PhoP, and PcrA proteins) and ATPase activity (GO:0016887) (including AddA, TagH, GrlA, Rho, PcrA, and MetN1 proteins) were predominant in $S$. epidermidis biofilm with prevented dormancy.

In order to analyze relative quantitation of label-free data, emPAI was compared among biological replicates. The reproducibility of data collected from the two biological replicates is shown in Fig. S2. Pearson's correlation indicated good reliability of emPAI data, showing a correlation higher than 0.810 in both conditions. Thus, we used emPAI average for further analysis. emPAI distribution among the two distinct biological conditions is shown in Fig. S3.

\section{Label-based data analyzes}

iTRAQ provide the possibility to quantitatively examine a large number of proteins for differential expression (Ross et al. 2004; Thompson et al. 2003). First, samples were labeled, pooled, fractionated by HPLC, and then separated by liquid chromatography and analyzed by tandem mass spectrometry (MS/MS). Using this approach, a total of 409 proteins were identified. Among them, 408 proteins had a calculated ratio value (distribution of iTRAQ ratio is shown in Fig. S4). Herein, a total of 29 proteins were differentially expressed, wherein 16 proteins were overexpressed and 13 proteins were underexpressed in $S$. epidermidis biofilms with prevented dormancy (Table S3). The two most represented biological processes, molecular functions, and KEGG pathways in differently expressed proteins were detailed in Fig. 4. Additionally to ion binding, GO terms related to catalytic activity, such as hydrolase (GO:0016810) (Atl and ArcA proteins), ligase (GO:0016874) (GlnA and GuaA proteins), and nucleotidyltransferase (GO:0016779) (RpoC and RpoB proteins) activity were associated with induced dormancy, since these GO terms were underexpressed. Purine (ser00230), arginine, and proline (ser00330) metabolism were the most prevalent pathways in underexpressed proteins in biofilms with prevented dormancy (Fig. S5). Conversely, molecular functions, such as structural constituent of ribosome (GO:0003735), structural molecule activity (GO:0005198) (including RpmI, RplL, RpmB, RplX, RplY, and RpsR proteins), and RNA binding (GO:0003723) were associated with proteins more expressed in $S$. epidermidis biofilm with prevented dormancy. Moreover, ribosome synthesis pathway 


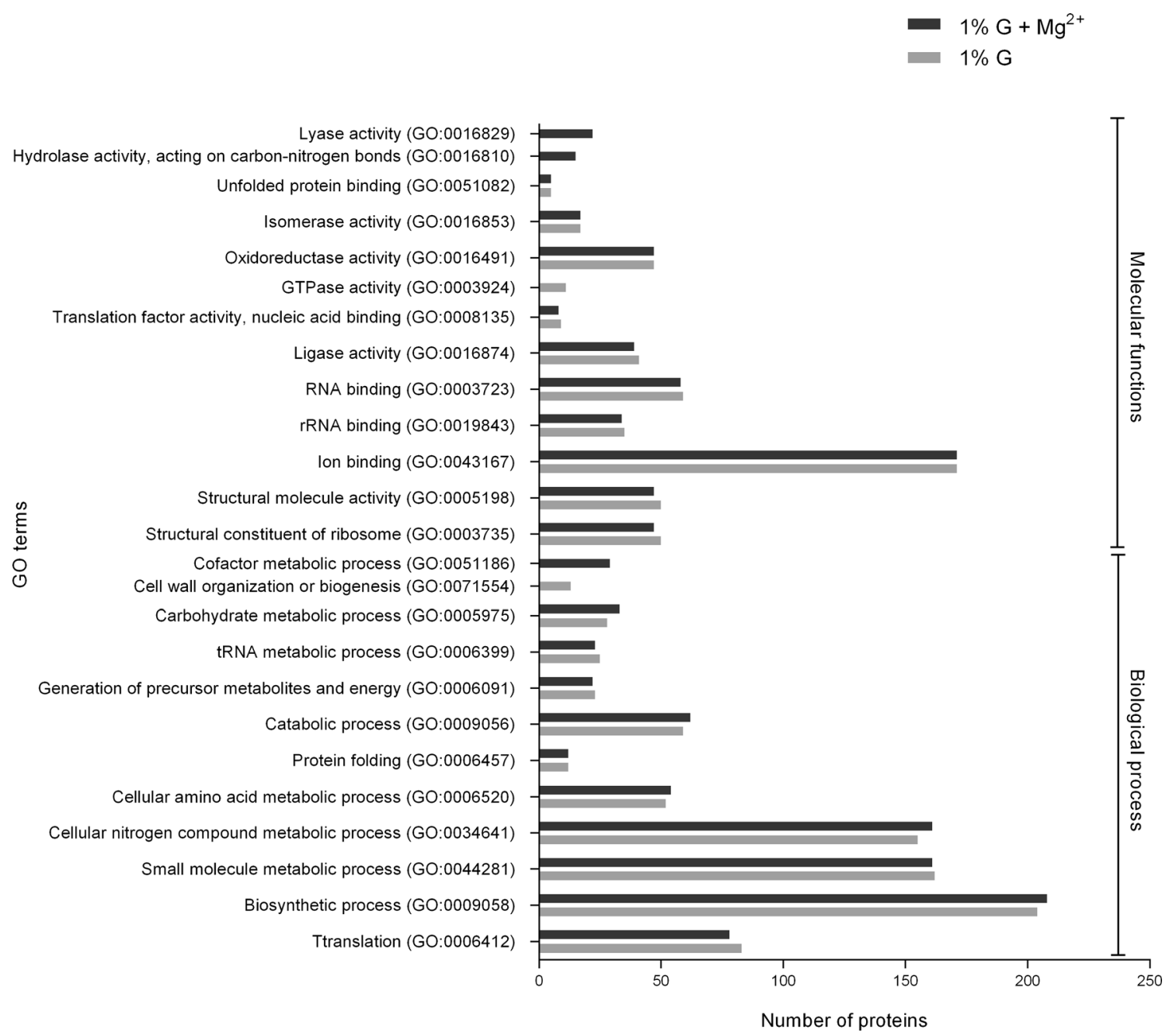

Fig. 2 Significant biological processes and molecular functions terms in S. epidermidis biofilms proteome with prevented $\left(1 \% \mathrm{G}+\mathrm{Mg}^{2+}\right)$ or induced $(1 \% \mathrm{G})$ dormancy $(p<0.05$, FDR-corrected). Data obtained by

(ser03010, including RpmI, RplL, RpmB, RplX, RplY, and RpsR proteins) was the most prevalent KEGG pathway in overexpressed proteins, which shows an involvement in translation process.

To elucidate the behavior of proteins which may be considered as Staphylococci virulence factors (Fey and Olson 2010; Otto 2012), we analyzed label-based data and list here their iTRAQ ratio: LipA $($ ratio $=1.13), \mathrm{ClpP}($ ratio $=$ $0.96), \mathrm{ClpC}($ ratio $=0.96), \mathrm{ClpB}(\mathrm{ratio}=0.93), \operatorname{PSM} \beta 1$ (ratio $=0.52), \operatorname{SitC}($ ratio $=0.80), \operatorname{AtlE}($ ratio $=0.70, p<0.05)$, TagD (ratio $=0.50)$, DltC (ratio $=0.45)$, and EbpS (ratio $=$ $2.3, p<0.05$ ). However, only AtlE (an autolysin, also involved in the attachment of $S$. epidermidis to abiotic surfaces (Heilmann et al. 1997)) and EbpS (primary attachment (Park et al. 1996)) had significant differences between induced and prevented dormancy, although these proteins did not present the same expression tendency. Interestingly, PSM $\beta 1$ (a noncytolytic modulin involved in in vitro detachment (Wang et al. 2011)) had a ratio of 0.52 but without label-free methodology. Classes with a $p$ value $\leq 0.05$ were considered statistically significant

statistical significance. Although differences were not statistically significant in the majority of proteins, virulence proteins seemed to be more expressed in biofilms with induced dormancy.

Search Tool for Interactions of Chemicals (STITCH v.4) allows the creation of a protein-chemical interaction network based on experimental data, databases, text mining, and predicted interactions (Kuhn et al. 2014). Since the growth model previously described includes addition of $\mathrm{Mg}^{2+}$ to obtain S. epidermidis biofilms with prevented dormancy (Cerca et al. 2011), STITCH resource was used to predict interactions of magnesium with unique proteins in prevented dormancy (identified by label-free) and overexpressed proteins (identified by label-based methodology) (accessed at December 10, 2014). Additionally, we applied the Markov cluster algorithm (MCL) to identify which proteins generate a cluster with magnesium (Fig. 5; complete network is shown in Fig. S6). Five of these proteins present nucleic acid binding function, and three of them are localized in cytoplasmic membrane. 
Fig. 3 The three most prevalent biological processes and molecular functions terms in proteins only found in S. epidermidis biofilms in prevented $\left(1 \% \mathrm{G}+\mathrm{Mg}^{2+}\right)$ or induced $(1 \% \mathrm{G})$ dormancy. Data obtained by label-free methodology

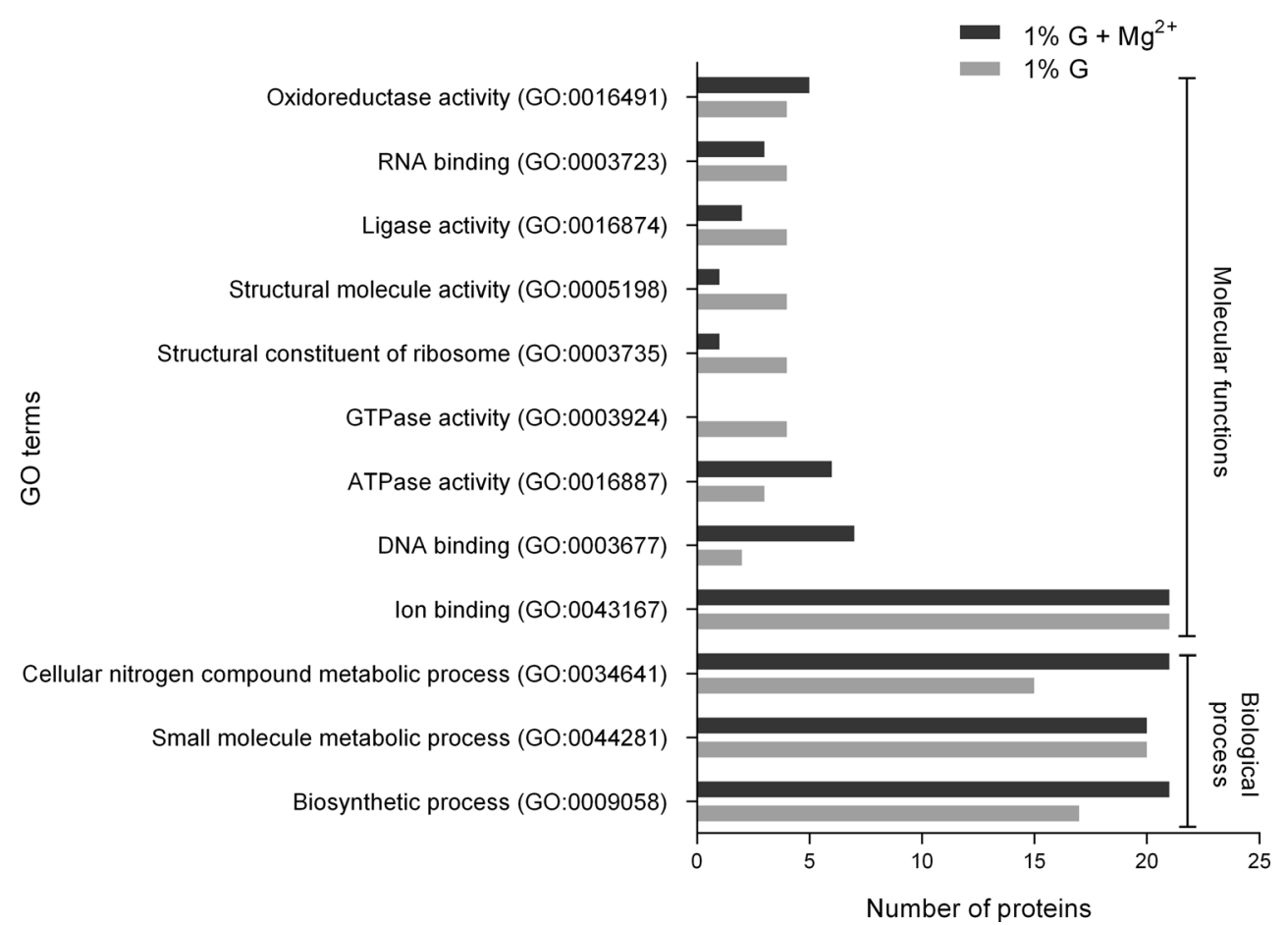

Protein susceptibility to carbonylation

Oxidative modification of proteins can assume many forms such as nitrotyrosination, carbonylation, methionine, and sulfhydryl oxidation (reviewed by Dean et al. (1997)). From those, protein carbonyl content is widely used as a marker for oxidative stress, since it is considered to be a major form of protein oxidation (Dalle-Donne et al. 2009). Previously, we compared the transcriptome of $S$. epidermidis biofilms with prevented and induced dormancy (Carvalhais et al. 2014), which suggested that oxidation-reduction molecular function was increased in $S$. epidermidis biofilms with higher number of dormant bacteria (Carvalhais et al. 2014). Following this, we evaluated protein oxidation in $S$. epidermidis biofilms with prevented and induced dormancy, namely protein carbonylation. A comparison of the 2-DE immunoblots reveals a different carbonylated pattern between prevented and induced dormancy (Fig. 6). Protein oxidation assessed by protein carbonyl content was mainly localized in the region around $\mathrm{p} I$ of 5 , with a molecular weight varying from 27 to $77 \mathrm{kDa}$. From the most
Fig. 4 The two most prevalent biological processes, molecular functions, and KEGG pathways in statistically significant overexpressed and underexpressed proteins from S. epidermidis biofilms with prevented dormancy. Data obtained by label-based methodology

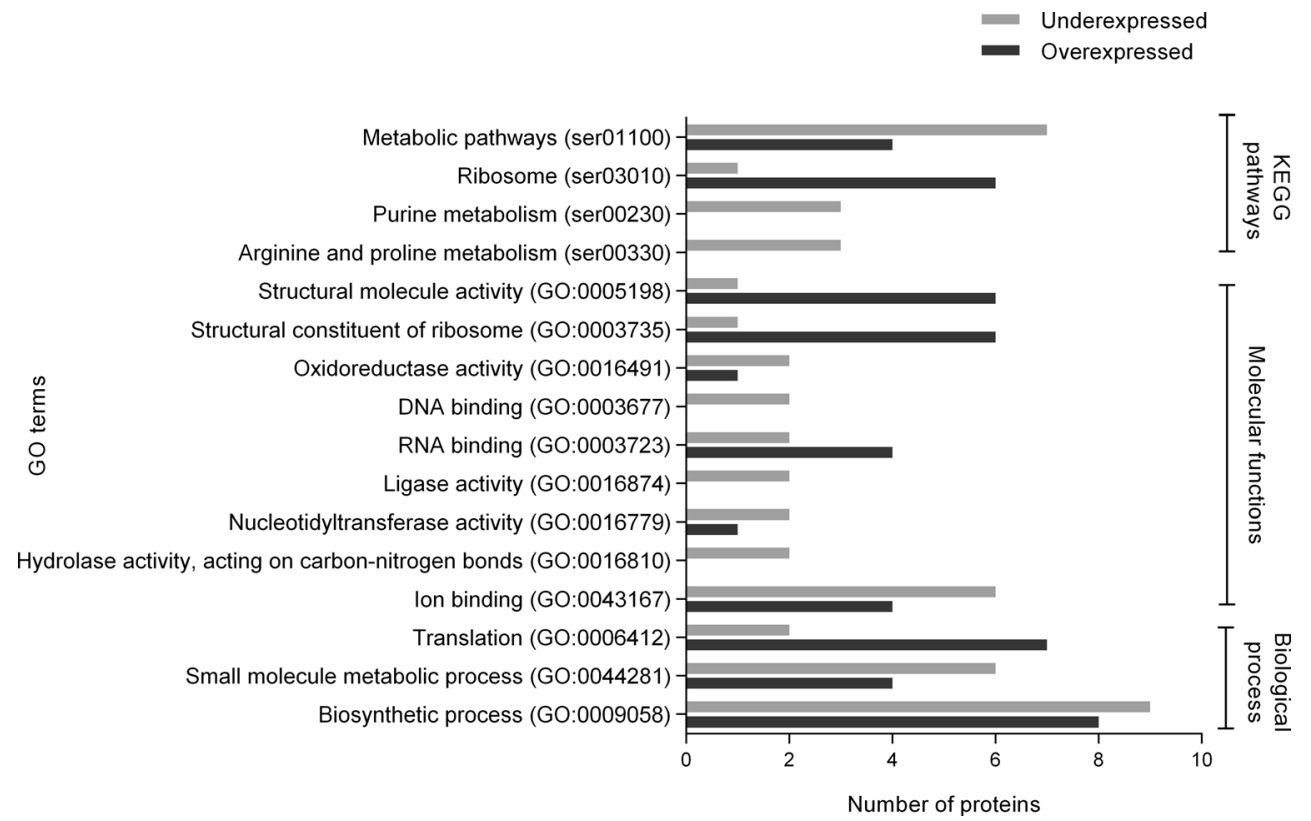




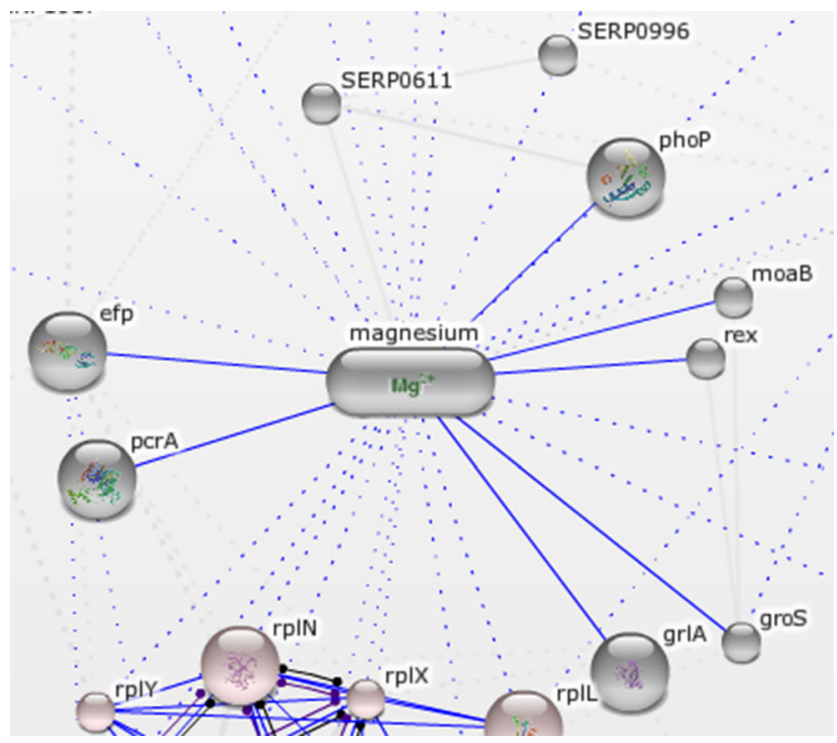

Fig. 5 Cluster of magnesium-protein interactions generated and predicted by STITCH (v.4). PhoP alkaline phosphatase synthesis transcriptional regulatory protein PhoP, Efp elongation factor P, PcrA ATP-dependent DNA helicase PcrA, MoaB molybdenum cofactor biosynthesis protein B; GroS 10 kDa chaperonin, GrlA DNA topoisomerase 4 subunit A, Rex redox-sensing transcriptional repressor Rex, SERP0996 probable CtpAlike serine protease, $S E R P 0611$ serine protease HtrA-like

susceptible proteins to oxidation identified in both conditions showed that TpiA and PfkA proteins seems to be associated to biofilm dormancy, whereas RpsA and Pgk were more reactive to DNP in S. epidermidis biofilms with prevented dormancy. A few more proteins presented the same oxidation susceptibility among the two tested conditions.

\section{Discussion}

Adaptation to local environment conditions contribute to physiological heterogeneity of bacteria within biofilms (Stewart and Franklin 2008). A subpopulation of dormant bacteria may be formed due to such unfavorable environment (Stewart and Franklin 2008). With a purpose of characterize dormancy within biofilms, a global scale analysis of transcriptome and proteome may be achieved with integration of highthroughput techniques and quantitative proteomics. Recently, we assessed transcriptome profile of $S$. epidermidis with prevented and induced dormancy (Carvalhais et al. 2014). However, similarly to other bacteria studies (reviewed by de Sousa et al. (2009)), comparison among S. epidermidis proteins and transcripts showed a modest correlation (Carvalhais et al. 2015). Currently, quantitative proteomics is still the bottleneck of comparative approaches, since the numbers of identified proteins are most of the times behind the expected numbers (Berghoff et al. 2013). Herein, two different proteomic approaches were used to quantify proteins of $S$. epidermidis

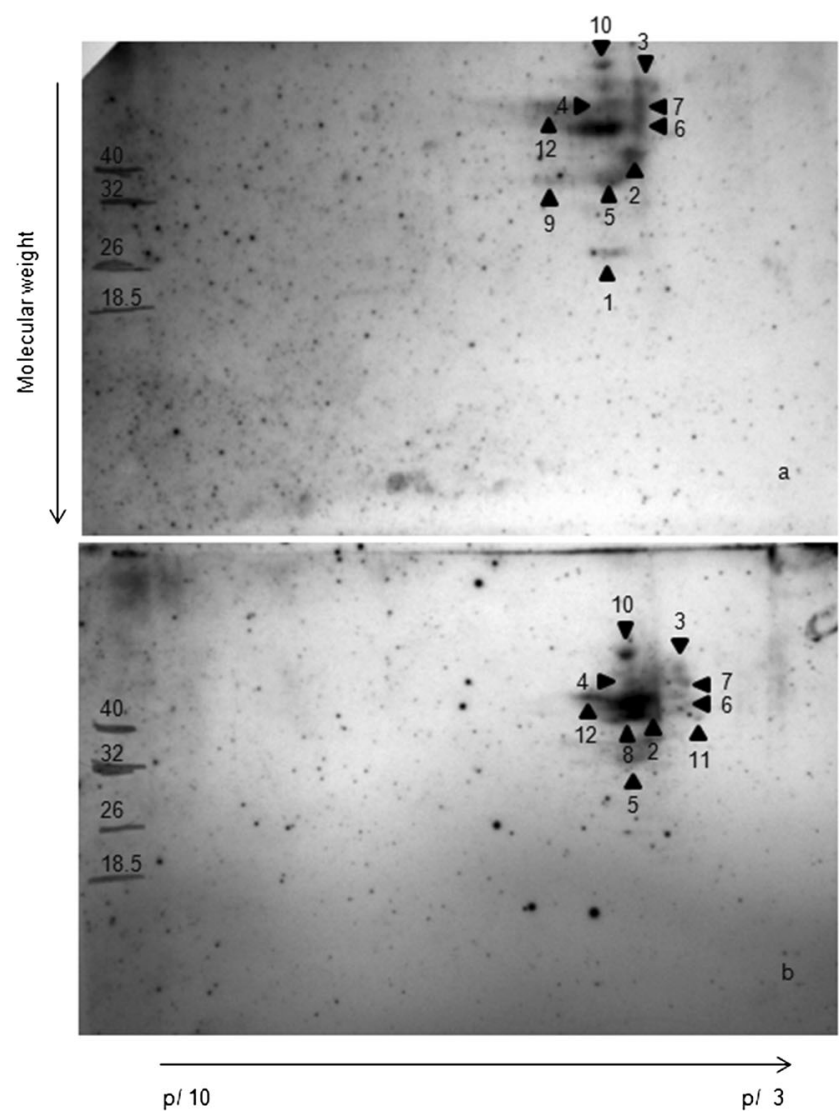

Fig. 6 Carbonylated proteins in induced (a) and prevented (b) dormancy. 1, triosephosphate isomerase (TpiA); 2, elongation factor Tu (EF-Tu); 3, chaperone protein DnaK (DnaK); 4, 2,3-bisphosphoglycerate-independent phosphoglycerate mutase (GpmI); 5, fructose-bisphosphate aldolase class 1 (Fda); 6, alkyl hydroperoxide reductase subunit F (AhpF); 7, $60 \mathrm{kDa}$ chaperonin (GroEL); 8, phosphoglycerate kinase (Pgk); 9, ATPdependent 6-phosphofructokinase (PfkA); 10, elongation factor $\mathrm{G}$ (FusA); 11, 30S ribosomal protein $\mathrm{S} 1$ (RpsA); 12, inosine-5'monophosphate dehydrogenase (GuaB)

biofilms, since iTRAQ methodology do not allow to identify unique protein in each condition, and it is used to validate protein expression data.

Based on label-free method, GO annotation strongly suggests the involvement of GTPase activity and ion-binding functions in dormancy within $S$. epidermidis biofilms (Fig. 2), since $\mathrm{Mg}^{2+}$ was used to avoid dormancy entry (Cerca et al. 2011). Furthermore, proteins only found in dormant condition had a high prevalence of GTPase activity (Fig. 3), which is in accordance with hydrolase activity of underexpressed proteins, found in label-based approach. GTPase enzymes are responsible for GTP hydrolysis and may be involved in several biological functions, from cell cycle regulation, tRNA modification, energy metabolism, stringent response, and stress response, among others (reviewed by Verstraeten et al. (2011)). Furthermore, in a nutrient limitation environment, GTP is converted by RelA (GTP pyrophosphokinase) to (p)ppGpp (guanosine $5^{\prime}$-triphosphate-3'-diphosphate and 5'-3'-bis-diphosphate) (Sonenshein 
2005; Verstraeten et al. 2011). RelA is a ribosome-associated (p)ppGpp synthetase and a hydrolase, which is activated in response to amino acid starvation (Cashel et al. 1996; Potrykus and Cashel 2008). Interestingly, RelA was among the uniquely expressed proteins in S. epidermidis biofilms with induced dormancy. Since in deeper biofilm layers bacteria have limited access to nutrients, this may increase their levels of (p)ppGpp (Maisonneuve et al. 2013), leading to higher expression of RelA protein. In the opposite, prevented dormancy condition presented enhancement of DNA binding and ATPase activity molecular functions, which is related to the higher number of metabolic active cells. Nowadays, it is assumed that toxin/antitoxin modules (TA) are involved in dormant persister cell formation (Keren et al. 2004). Currently, TA systems are assigned to five classes (I-V) according to their genetic structure and regulation (Goeders and Van Melderen 2014; Unterholzner et al. 2013). Increased expression of type I TA modules, such as TisB and HokE in E. coli, has ATP synthesis cellular process as a target (reviewed by Unterholzner et al. (2013)). Although not extensively studied, a type I TA module was already described in Staphylococcus aureus (Sayed et al. 2012). Additionally, the existence of other type I TA modules in S. aureus was predicted by bioinformatic tools but their biological activities were not confirmed (Fozo et al. 2010). The characterization of dormancy was also assessed by iTRAQ methodology as cross-check validation of protein expression data, which showed main differences in molecular functions, equivalently to label-free analysis (Fig. 4).

Proteomic analyses of dormant cells are limited (reviewed in Pinto et al. (2013)). Previous proteomic studies found a relation among GroEL (60 kDa chaperonin), DnaK (chaperone protein DnaK), AtpD (ATP synthase subunit beta), and Eno (enolase) proteins with viable but nonculturable state (VBNC) of bacteria, since their expression were lower (Heim et al. 2002). Also, a decreased expression of alkyl hydroperoxide reductase subunit $\mathrm{F}(\mathrm{AhpF})$ protein, an oxidationresponsive factor, was previously found in VBNC (Asakura et al. 2007). Our quantitative results showed that these proteins were decreased in dormancy, however without statistically significant difference. Additionally, EF-Tu protein (elongation factor $\mathrm{Tu}$ ) was also associated with $\mathrm{VBNC}$ but results are not consistent among studies (Pinto et al. 2013). Interestingly, here, EF-Tu showed a significant increased expression in dormancy. Similarly to transcriptomic profile of dormancy, overexpressed proteins were related to an enrichment of ribosome synthesis pathway and structural constituent of ribosome molecular function (Carvalhais et al. 2014). However, by a proteomic approach, we did not find a significant underexpression of proteins involved in oxidation-reduction, pyruvate metabolism, and acetyl-CoA metabolic processes. Nevertheless, we recently showed that correlation among transcripts and proteins were not strong in S. epidermidis biofilms
(Carvalhais et al. 2015). Thus, underexpressed proteins were mainly associated with purine and arginine and proline KEGG pathways. Ion binding and catalytic activity were the main molecular functions associated with underexpressed proteins in S. epidermidis biofilms with prevented dormancy (Fig. 4). Ligase, nucleotidyltransferase, and hydrolase activity combined with DNA and RNA binding may suggest that proteins less expressed in S. epidermidis biofilms with prevented dormancy are involved in transference of nucleotidyl groups and amino acid metabolism. Moreover, hydrolase and transferase protein classes have large number of cysteines, which are more susceptible to oxidation (Liebler 2008; Sanchez et al. 2008). Staphylococcus spp. use cysteine oxidation to respond to and overcome reactive oxygen species found in the host environment (Deng et al. 2013; Gaupp et al. 2012). Oxidative stress is described as a factor that contributes to persister cell increase (Hong et al. 2012). However, Leszczynska et al. found that persister cell frequency was not correlated with the oxidation of proteins (Leszczynska et al. 2013). Interestingly, here, we found that dormancy within S. epidermidis biofilms affected the pattern of oxidized proteins (Fig. 6). Among the carbonylated proteins, a few were related to oxidoreductase activity (such as GuaB and AhpF), with RNA binding (RpsA, EF-Tu, and FusA proteins), and others were involved in ATP binding (such as PfkA, Pgk, GroEL, and DnaK). Additionally, proteins involved in response to stress, DnaK and AhpF, were found carbonylated. Interestingly, among the carbonylated proteins, only EF-Tu, GpmI, and Pgk showed significant differences in protein expression between prevented and induced dormancy. Our results suggest that Pgk protein is more active in biofilms with prevented dormancy, but may be more susceptible to oxidation and may suffer biological modifications. However, future work in protein oxidation exploration may help to unravel if oxidation susceptibility may contribute as a factor that leads to dormancy.

It is known that magnesium is involved in several biological processes in bacteria, such as genomic stability, membrane stabilization, and cofactor in several enzymatic reactions (Groisman et al. 2013). Consistent with this fact, we found that proteins which formed a closer cluster with magnesium presented mainly nucleic acid binding function (Fig. 5). Interestingly, cytoplasmic membrane proteins were also found associated with magnesium action. Dunne et al. were the first to demonstrate the magnesium action over S. epidermidis adhesion (Dunne and Burd 1992). Earlier, Cutinelli and Galdiero aimed to describe the binding capacity of $\mathrm{Mg}^{2+}$, among other ions, to $S$. aureus cell wall (Cutinelli and Galdiero 1967). They proposed that high $\mathrm{pH}$ values increased the amount of $\mathrm{Mg}^{2+}$ bound to cell wall. Since we previously shown that $\mathrm{pH}$ does not vary between the two growth conditions (Carvalhais et al. 2014), this statement cannot explain the $\mathrm{Mg}^{2+}$ binding in the present model. Similarly, 
$\mathrm{Mg}^{2+}$ affinity to Bacillus subtilis cell wall was influenced by the presence of functional groups in the peptidoglycan matrix (Doyle et al. 1980). In addition, Piddington and colleagues demonstrated that $\mathrm{Mg}^{2+}$ is essential to Mycobacterium tuberculosis growth in acidic conditions and it could not be replaced by other divalent cations (Piddington et al. 2000). Also, a possible influence of $\mathrm{Mg}^{2+}$ in protein structure and physical properties was also described in M. tuberculosis (Bhatt et al. 2005). Recently, it was shown that catheters coated with magnesium fluoride nanoparticles presented a significantly reduction of $S$. aureus and Escherichia coli colonization (Lellouche et al. 2012). However, the $\mathrm{Mg}^{2+}$ effect in biofilm maturation and its role on dormancy remains poorly understood (Piddington et al. 2000; Song and Leff 2006).

To the best of our knowledge, the present study represents the first attempt to determine protein expression differences associated to dormancy within biofilms. For the first time, quantitative proteomics was performed in order to assess dormancy within S. epidermidis biofilms. Label-free and labelbased methods afforded similar and relevant biological data of dormancy physiological state. Overexpressed proteins were mainly related to ribosome synthesis pathways. Conversely, based on our dormancy model, ion binding and catalytic activity molecular functions seem to be related to the absence of magnesium enrichment, which contribute to dormancy entrance. It should also be emphasized that the output of the different quantitative strategies here employed, contributed to a further exploration of dormant physiological condition.

Acknowledgements This work was funded by Fundação para a Ciência e a Tecnologia (FCT) and COMPETE grants PTDC/BIA-MIC/ 113450/2009, FCOMP-01-0124-FEDER-014309, QOPNA research unit (project PEst-C/QUI/UI0062/2013), RNEM (National Mass Spectrometry Network), and CENTRO-07-ST24-FEDER-002034. The authors also thank the FCT Strategic Project PEst-OE/EQB/LA0023/2013, the Project NORTE-07-0124-FEDER-000027, co-funded by the Programa Operacional Regional do Norte (ON.2 - O Novo Norte), QREN, FEDE $\mathrm{R}$, and the project RECI/EBB-EBI/0179/2012, FCOMP-01-0124FEDER-027462. VC has an individual FCT fellowship (SFRH/BD/ 78235/2011). NC is an Investigator FCT.

\section{References}

Abdallah C, Dumas-Gaudot E, Renaut J, Sergeant K (2012) Gel-based and gel-free quantitative proteomics approaches at a glance. Int $\mathrm{J}$ Plant Genomics 2012:494-572

Alves RM, Vitorino R, Padrao AI, Moreira-Goncalves D, Duarte JA, Ferreira RM, Amado F (2013) iTRAQ-based quantitative proteomic analysis of submandibular glands from rats with STZ-induced hyperglycemia. J Biochem 153:209-220

Asakura H, Panutdaporn N, Kawamoto K, Igimi S, Yamamoto S, Makino S (2007) Proteomic characterization of enterohemorrhagic Escherichia coli $\mathrm{O} 157: \mathrm{H} 7$ in the oxidation-induced viable but non-culturable state. Microbiol Immunol 51:875-881

Ashburner M, Ball CA, Blake JA, Botstein D, Butler H, Cherry JM, Davis AP, Dolinski K, Dwight SS, Eppig JT, Harris MA, Hill DP,
Issel-Tarver L, Kasarskis A, Lewis S, Matese JC, Richardson JE, Ringwald M, Rubin GM, Sherlock G (2000) Gene ontology: tool for the unification of biology. The Gene Ontology Consortium. Nat Genet 25:25-29

Balaban NQ, Gerdes K, Lewis K, McKinney JD (2013) A problem of persistence: still more questions than answers? Nat Rev Microbiol 11:587-591

Berghoff BA, Konzer A, Mank NN, Looso M, Rische T, Forstner KU, Kruger M, Klug G (2013) Integrative "omics"-approach discovers dynamic and regulatory features of bacterial stress responses. PLoS Genet 9, e1003576

Bhatt AN, Shukla N, Aliverti A, Zanetti G, Bhakuni V (2005) Modulation of cooperativity in Mycobacterium tuberculosis $\mathrm{NADPH}-$ ferredoxin reductase: cation-and $\mathrm{pH}$-induced alterations in native conformation and destabilization of the $\mathrm{NADP}^{+}$-binding domain. Protein Sci 14:980-992

Cain JA, Solis N, Cordwell SJ (2013) Beyond gene expression: the impact of protein post-translational modifications in bacteria. $\mathrm{J}$ Proteomics 97:265-286

Carvalhais V, Franca A, Cerca F, Vitorino R, Pier GB, Vilanova M, Cerca N (2014) Dormancy within Staphylococcus epidermidis biofilms: a transcriptomic analysis by RNA-seq. Appl Microbiol Biotechnol 98:2585-2596

Carvalhais V, Franca A, Pier GB, Vilanova M, Cerca N, Vitorino R (2015) Comparative proteomic and transcriptomic profile of Staphylococcus epidermidis biofilms grown in glucose-enriched medium. Talanta 132:705-712

Cashel M, Gentry D, Hernandez V, Vinella D (1996) The stringent response. In: Neidhardt FC, Curtiss IIIR, Ingraham JL, Lin ECC, Low KB, Magasanik B, Reznikoff WS, Riley M, Schaechter M, Umbarger HE (ed) Escherichia coli and Salmonella: Cellular and Molecular Biology, 2nd edn. AMS Press, Washington DC, pp 14581496

Cerca N, Martins S, Cerca F, Jefferson KK, Pier GB, Oliveira R, Azeredo J (2005) Comparative assessment of antibiotic susceptibility of coagulase-negative staphylococci in biofilm versus planktonic culture as assessed by bacterial enumeration or rapid XTT colorimetry. J Antimicrob Chemother 56:331-336

Cerca N, Jefferson KK, Oliveira R, Pier GB, Azeredo J (2006) Comparative antibody-mediated phagocytosis of Staphylococcus epidermidis cells grown in a biofilm or in the planktonic state. Infect Immun 74:4849-4855

Cerca F, Andrade F, Franca A, Andrade EB, Ribeiro A, Almeida AA, Cerca N, Pier G, Azeredo J, Vilanova M (2011) Staphylococcus epidermidis biofilms with higher proportions of dormant bacteria induce a lower activation of murine macrophages. J Med Microbiol 60:1717-1724

Cerca F, Franca A, Perez-Cabezas B, Carvalhais V, Ribeiro A, Azeredo J, Pier GB, Cerca N, Vilanova M (2014) Dormant bacteria within Staphylococcus epidermidis biofilms have low inflammatory properties and maintain tolerance to vancomycin and penicillin after entering planktonic growth. J Med Microbiol 63:1274-1283

Conrad CC, Choi J, Malakowsky CA, Talent JM, Dai R, Marshall P, Gracy RW (2001) Identification of protein carbonyls after twodimensional electrophoresis. Proteomics 1:829-834

Cucarella C, Solano C, Valle J, Amorena B, Lasa I, Penades JR (2001) Bap, a Staphylococcus aureus surface protein involved in biofilm formation. J Bacteriol 183:2888-2896

Cutinelli C, Galdiero F (1967) Ion-binding properties of the cell wall of Staphylococcus aureus. J Bacteriol 93:2022-2023

Dalle-Donne I, Carini M, Orioli M, Vistoli G, Regazzoni L, Colombo G, Rossi R, Milzani A, Aldini G (2009) Protein carbonylation: 2,4dinitrophenylhydrazine reacts with both aldehydes/ketones and sulfenic acids. Free Radic Biol Med 46:1411-1419 
de Sousa AR, Penalva LO, Marcotte EM, Vogel C (2009) Global signatures of protein and mRNA expression levels. Mol BioSyst 5:1512 1526

Dean RT, Fu S, Stocker R, Davies MJ (1997) Biochemistry and pathology of radical-mediated protein oxidation. Biochem J 324:1-18

Deng X, Weerapana E, Ulanovskaya O, Sun F, Liang H, Ji Q, Ye Y, Fu Y, Zhou L, Li J, Zhang H, Wang C, Alvarez S, Hicks LM, Lan L, Wu M, Cravatt BF, He C (2013) Proteome-wide quantification and characterization of oxidation-sensitive cysteines in pathogenic bacteria. Cell Host Microbe 13:358-370

Donlan RM (2001) Biofilms and device-associated infections. Emerg Infect Dis 7:277-281

Dorr T, Vulic M, Lewis K (2010) Ciprofloxacin causes persister formation by inducing the TisB toxin in Escherichia coli. PLoS Biol 8, e1000317

Doyle RJ, Matthews TH, Streips UN (1980) Chemical basis for selectivity of metal ions by the Bacillus subtilis cell wall. J Bacteriol 143: 471-480

Dunne v Jr, Burd EM (1992) The effects of magnesium, calcium, EDTA, and $\mathrm{pH}$ on the in vitro adhesion of Staphylococcus epidermidis to plastic. Microbiol Immunol 36:1019-1027

Fey PD (2010) Modality of bacterial growth presents unique targets: how do we treat biofilm-mediated infections? Curr Opin Microbiol 13: 610-615

Fey PD, Olson ME (2010) Current concepts in biofilm formation of Staphylococcus epidermidis. Future Microbiol 5:917-933

Fozo EM, Makarova KS, Shabalina SA, Yutin N, Koonin EV, Storz G (2010) Abundance of type I toxin-antitoxin systems in bacteria: searches for new candidates and discovery of novel families. Nucleic Acids Res 38:3743-3759

Franceschini A, Szklarczyk D, Frankild S, Kuhn M, Simonovic M, Roth A, Lin J, Minguez P, Bork P, von Mering C, Jensen LJ (2013) STRI NG v9.1: protein-protein interaction networks, with increased coverage and integration. Nucleic Acids Res 41:D808-D815

Gaupp R, Ledala N, Somerville GA (2012) Staphylococcal response to oxidative stress. Front Cell Infect Microbiol 2:33

Goeders N, Van Melderen L (2014) Toxin-antitoxin systems as multilevel interaction systems. Toxins (Basel) 6:304-324

Groisman EA, Hollands K, Kriner MA, Lee EJ, Park SY, Pontes MH (2013) Bacterial $\mathrm{Mg}^{2+}$ homeostasis, transport, and virulence. Annu Rev Genet 47:625-646

Guell M, Yus E, Lluch-Senar M, Serrano L (2011) Bacterial transcriptomics: what is beyond the RNA horiz-ome? Nat Rev Microbiol 9: 658-669

Hall-Stoodley L, Costerton JW, Stoodley P (2004) Bacterial biofilms: from the natural environment to infectious diseases. Nat Rev Microbiol 2:95-108

Heilmann C, Hussain M, Peters G, Gotz F (1997) Evidence for autolysinmediated primary attachment of Staphylococcus epidermidis to a polystyrene surface. Mol Microbiol 5:1013-1024

Heim S, Lleo M, Bonato B, Guzman CA, Canepari P (2002) The viable but nonculturable state and starvation are different stress responses of Enterococcus faecalis, as determined by proteome analysis. J Bacteriol 184:6739-6745

Hong SH, Wang X, O'Connor HF, Benedik MJ, Wood TK (2012) Bacterial persistence increases as environmental fitness decreases. Microb Biotechnol 5:509-522

Ishihama Y, Oda Y, Tabata T, Sato T, Nagasu T, Rappsilber J, Mann M (2005) Exponentially modified protein abundance index (emPAI) for estimation of absolute protein amount in proteomics by the number of sequenced peptides per protein. Mol Cell Proteomics 4:1265-1272

Kanehisa M, Goto S, Kawashima S, Okuno Y, Hattori M (2004) The KEGG resource for deciphering the genome. Nucleic Acids Res 32:D277-D280

Kaprelyants AS, Gottschal JC, Kell DB (1993) Dormancy in nonsporulating bacteria. FEMS Microbiol Rev 10:271-285
Keren I, Shah D, Spoering A, Kaldalu N, Lewis K (2004) Specialized persister cells and the mechanism of multidrug tolerance in Escherichia coli. J Bacteriol 186:8172-8180

Kuhn M, Szklarczyk D, Pletscher-Frankild S, Blicher TH, von Mering C, Jensen LJ, Bork P (2014) STITCH 4: integration of protein-chemical interactions with user data. Nucleic Acids Res 42:D401-D407

Lasa I, Penades JR (2006) Bap: a family of surface proteins involved in biofilm formation. Res Microbiol 157:99-107

Lellouche J, Friedman A, Lahmi R, Gedanken A, Banin E (2012) Antibiofilm surface functionalization of catheters by magnesium fluoride nanoparticles. Int J Nanomedicine 7:1175-1188

Leszczynska D, Matuszewska E, Kuczynska-Wisnik D, FurmanekBlaszk B, Laskowska E (2013) The formation of persister cells in stationary-phase cultures of Escherichia coli is associated with the aggregation of endogenous proteins. PLoS One 8, e54737

Lewis K (2007) Persister cells, dormancy and infectious disease. Nat Rev Microbiol 5:48-56

Liebler DC (2008) Protein damage by reactive electrophiles: targets and consequences. Chem Res Toxicol 21:117-128

Mack D, Siemssen N, Laufs R (1992) Parallel induction by glucose of ahderence and a polysaccharide antigen specific for plastic-adherent Staphylococcus epidermidis: evidence for functional relation to intercellular adhesion. Infect Immun 60:2048-2057

Maisonneuve E, Castro-Camargo M, Gerdes K (2013) (p)ppGpp controls bacterial persistence by stochastic induction of toxin-antitoxin activity. Cell 154:1140-1150

Oliveros JC (2007) VENNY. An interactive tool for comparing lists with Venn Diagrams. http://bioinfogp.cnb.csic.es/tools/venny/

Orman MA, Brynildsen MP (2013) Dormancy is not necessary or sufficient for bacterial persistence. Antimicrob Agents Chemother 57:3230-3239

Otto M (2012) Molecular basis of Staphylococcus epidermidis infections. Semin Immunopathol 34:201-214

Otto M (2013) Staphylococcal infections: mechanisms of biofilm maturation and detachment as critical determinants of pathogenicity. Annu Rev Med 64:175-188

Otto M (2014) Staphylococcus epidermidis pathogenesis. Methods Mol Biol 1106:17-31

Park PW, Rosenbloom J, Abrams WR, Rosenbloom J, Mecham RP (1996) Molecular cloning and expression of the gene for elastin-binding protein (ebpS) in Staphylococcus aureus. J Biol Chem 271:15803-15809

Piddington DL, Kashkouli A, Buchmeier NA (2000) Growth of Mycobacterium tuberculosis in a defined medium is very restricted by acid $\mathrm{pH}$ and $\mathrm{Mg}^{2+}$ levels. Infect Immun 68:4518-4522

Pinto D, Santos MA, Chambel L (2013) Thirty years of viable but nonculturable state research: Unsolved molecular mechanisms. Crit Rev Microbiol 41:61-76

Potrykus K, Cashel M (2008) (p)ppGpp: still magical? Annu Rev Microbiol 62:35-51

Qin Z, Ou Y, Yang L, Zhu Y, Tolker-Nielsen T, Molin S, Qu D (2007) Role of autolysin-mediated DNA release in biofilm formation of Staphylococcus epidermidis. Microbiology 153:2083-2092

Robinson CE, Keshavarzian A, Pasco DS, Frommel TO, Winship DH, Holmes EW (1999) Determination of protein carbonyl groups by immunoblotting. Anal Biochem 266:48-57

Rohde H, Burdelski C, Bartscht K, Hussain M, Buck F, Horstkotte MA, Knobloch JK, Heilmann C, Herrmann M, Mack D (2005) Induction of Staphylococcus epidermidis biofilm formation via proteolytic processing of the accumulation-associated protein by staphylococcal and host proteases. Mol Microbiol 55:1883-1895

Ross PL, Huang YN, Marchese JN, Williamson B, Parker K, Hattan S, Khainovski N, Pillai S, Dey S, Daniels S, Purkayastha S, Juhasz P, Martin S, Bartlet-Jones M, He F, Jacobson A, Pappin DJ (2004) Multiplexed protein quantitation in Saccharomyces cerevisiae using amine-reactive isobaric tagging reagents. Mol Cell Proteomics 3: $1154-1169$ 
Sadovskaya I, Vinogradov E, Li J, Jabbouri S (2004) Structural elucidation of the extracellular and cell-wall teichoic acids of Staphylococcus epidermidis RP62A, a reference biofilm-positive strain. Carbohydr Res 339:1467-1473

Sadovskaya I, Vinogradov E, Flahaut S, Kogan G, Jabbouri S (2005) Extracellular carbohydrate-containing polymers of a model biofilm-producing strain, Staphylococcus epidermidis RP62A. Infect Immun 73:3007-3017

Sanchez R, Riddle M, Woo J, Momand J (2008) Prediction of reversibly oxidized protein cysteine thiols using protein structure properties. Protein Sci 17:473-481

Sayed N, Nonin-Lecomte S, Rety S, Felden B (2012) Functional and structural insights of a Staphylococcus aureus apoptotic-like membrane peptide from a toxin-antitoxin module. J Biol Chem 287: 43454-43463

Sonenshein AL (2005) CodY, a global regulator of stationary phase and virulence in Gram-positive bacteria. Curr Opin Microbiol 8:203207

Song B, Leff LG (2006) Influence of magnesium ions on biofilm formation by Pseudomonas fluorescens. Microbiol Res 161:355-361

Stewart PS, Franklin MJ (2008) Physiological heterogeneity in biofilms. Nat Rev Microbiol 6:199-210

Straub L (2011) Beyond the transcripts: what controls protein variation? PLoS Biol 9, e1001146

Thompson A, Schafer J, Kuhn K, Kienle S, Schwarz J, Schmidt G, Neumann T, Johnstone R, Mohammed AK, Hamon C (2003) Tandem mass tags: a novel quantification strategy for comparative analysis of complex protein mixtures by MS/MS. Anal Chem 75: 1895-1904
Unterholzner SJ, Poppenberger B, Rozhon W (2013) Toxin-antitoxin systems: Biology, identification, and application. Mob Genet Elements 3, e26219

Verstraeten N, Fauvart M, Versees W, Michiels J (2011) The universally conserved prokaryotic GTPases. Microbiol Mol Biol Rev 75:507542

Vitorino R, Guedes S, Manadas B, Ferreira R, Amado F (2012) Toward a standardized saliva proteome analysis methodology. J Proteomics 75:5140-5165

Vuong C, Kocianova S, Voyich JM, Yao Y, Fischer ER, DeLeo FR, Otto M (2004) A crucial role for exopolysaccharide modification in bacterial biofilm formation, immune evasion, and virulence. J Biol Chem 279:54881-54886

Wakamoto Y, Dhar N, Chait R, Schneider K, Signorino-Gelo F, Leibler S, McKinney JD (2013) Dynamic persistence of antibiotic-stressed mycobacteria. Science 339:91-95

Wang R, Khan BA, Cheung GY, Bach TH, Jameson-Lee M, Kong KF, Queck SY, Otto M (2011) Staphylococcus epidermidis surfactant peptides promote biofilm maturation and dissemination of biofilmassociated infection in mice. J Clin Invest 121:238-248

Williamson KS, Richards LA, Perez-Osorio AC, Pitts B, McInnerney K, Stewart PS, Franklin MJ (2012) Heterogeneity in Pseudomonas aeruginosa biofilms includes expression of ribosome hibernation factors in the antibiotic-tolerant subpopulation and hypoxiainduced stress response in the metabolically active population. J Bacteriol 194:2062-2073

Zhang W, Li F, Nie L (2010) Integrating multiple 'omics' analysis for microbial biology: application and methodologies. Microbiology 156:287-301 